\title{
BMJ Open Patch validation: an observational study protocol for the evaluation of a multisignal wearable sensor in patients during anaesthesia and in the postanaesthesia care unit
}

\author{
Morgan Le Guen, ${ }^{1}$ Pierre Squara (D) ,2 Sabrina Ma, ${ }^{1}$ Shérifa Adjavon, ${ }^{1}$ \\ Bernard Trillat, ${ }^{3}$ Messaouda Merzoug, ${ }^{4}$ Philippe Aegerter, ${ }^{5,6}$ Marc Fischler (1) ${ }^{1}$
}

To cite: Le Guen M, Squara P, Ma S, et al. Patch validation: an observational study protocol for the evaluation of a multisignal wearable sensor in patients during anaesthesia and in the postanaesthesia care unit. BMJ Open 2020;10:e040453. doi:10.1136/ bmjopen-2020-040453

- Prepublication history for this paper is available online. To view these files, please visit the journal online (http://dx.doi. org/10.1136/bmjopen-2020040453).

Received 20 May 2020

Revised 21 July 2020

Accepted 04 September 2020

Check for updates

(C) Author(s) (or their employer(s)) 2020. Re-use permitted under CC BY-NC. No commercial re-use. See rights and permissions. Published by BMJ.

For numbered affiliations see end of article.

Correspondence to Professor Marc Fischler; m.fischler@orange.fr

\section{ABSTRACT}

Introduction Except for operating rooms, postanaesthesia care units and intensive care units, where the monitoring of vital signs is continuous, intermittent care is standard practice. However, at a time when only the patients with the most serious conditions are hospitalised and only a fraction of these patients are in intensive care units, this type of monitoring is no longer sufficient. Wireless monitoring has been proposed, but it requires rigorous validation. The aim of this observational study is to compare vital signs obtained from a precordial patch sensor to those obtained with conventional monitoring. Methods and analysis This patch validation trial will be an observational, prospective, single-centre open study of 115 anaesthetised adult patients monitored with both a wireless sensor (myAngel VitalSigns, Devinnova, Montpellier, France) and a standard bedside monitor (Carescape Monitor B850, GE Healthcare, Chicago, Illinois). Both sensors will be used to record peripheral oxygen saturation, respiratory rate, heart rate, body temperature and blood pressure (systolic and diastolic). The main objective will be to assess the degree of agreement between the two systems during the patients' stay in the postanaesthesia care unit, both at the raw signal level and at the clinical parameter level. The secondary objectives will be to assess the same performance under anaesthesia, the frequency of missing data or artefacts, the diagnostic performance of the systems, the influence of patients' characteristics on agreement between the two systems, the adverse events and the acceptability of the patch to patients. Bland-Altman plots will be used in the main analysis to detect discrepancies and estimate the limits of agreement.

Ethics and dissemination Ethics approval was obtained from the Ethical Committee (Toulouse, France) on 10 April 2020. We are not yet recruiting subjects for this study. The results will be submitted for publication in peer-reviewed journals.

Trial registration number NCT04344093.

\section{INTRODUCTION}

Although some surgical patients with severe comorbidities or complications are

\section{Strengths and limitations of this study}

- This is the first study to validate a new multisignal wearable sensor in patients when they are under anaesthesia and in the postanaesthesia care unit (PACU), which are times when signal artefacts commonly occur.

- The study results will help determine the level of agreement between the parameters collected by a conventional monitor and the patch (in particular, blood pressure, heart rate, respiratory rate, oxygen saturation and temperature).

- The study results will also estimate the frequency of artefacts and determine the acceptability of this patch to patients during their stay in the PACU.

- Validation of this device during anaesthesia and PACU stay could not however be generalised to the postoperative period in the ward where patients are more mobile with an increased risk of artefacts.

hospitalised in units with a high level of monitoring, most patients are hospitalised in conventional units where clinical supervision is infrequent, particularly during the night. ${ }^{1}$

Adverse events occur frequently after surgery, as shown by a prospective international 7-day cohort study of outcomes following elective adult inpatient surgery in 44814 patients in 27 countries. A total of 7508 patients $(16.8 \%)$ developed one or more postoperative complications, and 207 patients $(0.5 \%)$ died. $^{2}$ Hospital costs are significantly increased by these complications, ${ }^{3}$ which are largely due to the inability to quickly detect significant worsening of a patient's condition. ${ }^{4}$

To improve nurses' ability to assess a patient's clinical situation, the Early Warning Score (EWS) is measured repeatedly. This score initially included five physiological 
parameters: heart rate, systolic blood pressure, respiratory rate, temperature and consciousness level. ${ }^{5}$ Many variants that include additional variables, such as oxygen saturation, urine output and clinical signs of deterioration (pallor, sweating, looking unwell), have been proposed. National Health Service England promoted the adoption of the National Early Warning Score 2 for adult patients by March 2019. ${ }^{6}$ However, studies have shown contradictory results regarding the added value of the EWS in relation to patient outcomes. ${ }^{8-10}$

Alternatively, clinical evaluation by nurses can be augmented by devices that allow the continuous monitoring of vital signs. Towards this end, Philips General Care Solutions proposed an automated Modified Early Warning Score monitoring system, the Philips IntelliVue Guardian Solution (Guardian), ${ }^{11}$ and concerning wearable vital sign monitoring devices, Weenk et alshowed that the ViSi Mobile and the HealthPatch give more frequent alerts than do nurses. ${ }^{12}$ Michard et al reviewed numerous innovations, particularly those designed to detect respiratory complications using wearable and wireless sensors. ${ }^{13}$ Before new sensors can be used, their accuracy and reliability must be verified. ${ }^{14}{ }^{15}$ Validation is of great importance, since the general public can buy lay user devices that seem similar but do not yield high-quality results. For example, Gillinov et al compared five optical heart rate monitors during various types of aerobic exercise and showed large differences between the monitors and a reference (an electrocardiograph device). ${ }^{16}$ van Lier et al recently reported at least three major reasons for inadequate validation (the use of different and sometimes inappropriate statistical methods, the evaluation of different levels for each parameter and a lack of criteria to determine validity) and recently published a standardised protocol for assessing the validity of physiological signals from wearable technology. ${ }^{17}$

Many portable wireless monitoring devices, measuring various numbers of physiological parameters, have been subjected to validation studies. ${ }^{18} 19$ We have focused our interest on a new device, the myAngel VitalSigns (VS), which is a multimodal medical device including three electrocardiography (ECG) leads and sensors that can measure physiological parameters such as blood pressure, heart rate, respiratory rate, peripheral oxygen saturation $\left(\mathrm{SpO}_{2}\right)$, actimetry, posture and body temperature. We aim to evaluate this device, which has never been validated or used previously, during patients' postanaesthesia care unit (PACU) stays (main objective), when their movements can generate artefacts, and during surgical procedures (one of the secondary objectives), when electrocautery and electronic devices can also create artefacts.

\section{METHODS AND ANALYSIS}

\section{Trial design}

This prospective observational study will be conducted in an academic hospital in which all types of surgical procedures except cardiac and orthopaedic procedures are

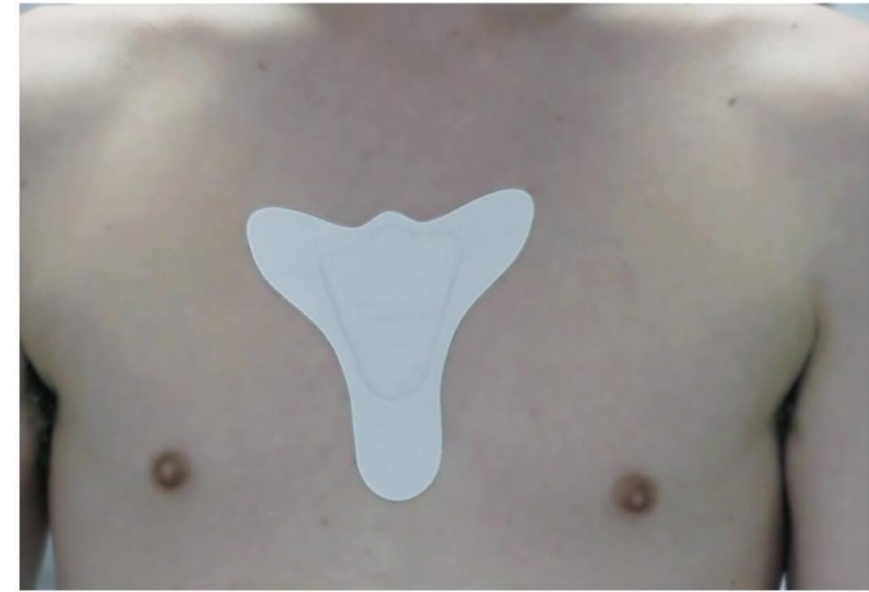

Figure 1 Placement of the patch sensor.

performed. The study has not yet recruited patients. They will be consecutively enrolled and followed up for their entire stay in the operating room and the PACU.

\section{Participant eligibility and consent}

Patients will be included if they meet all of the following criteria: (1) over 18 and under 85 years of age, (2) general anaesthesia for extrathoracic surgery, (3) supine position during surgery, and (4) written informed consent. The exclusion criteria are as follows: (1) pregnant or breastfeeding women, (2) patients with previous severe skin reactions to adhesives, and (3) patients deprived of liberty or under guardianship.

\section{The studied device}

The VS medical device, which has not yet obtained European Community or Food and Drug Administration approval, comprises a reusable electronic module, which allows physiological data to be acquired, and a disposable skin patch, which secures three contact electrodes (figure 1; Devinnova). Sensors integrated into the electronic module allow the measurement of the following vital parameters: three-lead ECG signals, oxygen saturation, respiratory rate, heart rate, body temperature, blood pressure (systolic and diastolic), actimetry (distance travelled, speed, number of steps and posture) and abrupt changes in position (impacts, falls). The electrodes enable signals to be measured from three ECG leads (DI, augmented vector left and augmented vector right). The patch sensor also includes a dry zinc/air battery (button cell, $1.4 \mathrm{~V}, 900 \mathrm{mAh}$ ), which powers the electronic module and allows the medical device to function for up to 5 days.

The heart rate measurements are based on the detection of R peaks, enabling RR intervals to be analysed. Blood pressure is determined from the pulse transit time and is calculated by proprietary and artificial intelligence methods. ${ }^{20-22}$ Respiratory rate is measured from a pressure sensor that evaluates the variation in chest signal amplitude from a sealed chamber at a constant pressure. Oxygen saturation is measured by an infrared transceiver that maintains constant and homogeneous contact at the 
emitted wavelength; the reflection measurements make this method reproducible and reliable. ${ }^{23}$ The temperature is measured by infrared spectroscopy, which also has high reproducibility over time. A six-axis accelerometer is used to evaluate the gravitational effect, that is, a patient's postural position (lying down, resting on the right or left flank, standing, sitting, immobile, moving) as well as actimetry (number of steps, distance covered, speed) and fall detection. The patch is waterproof, and its size is appropriate for daily use.

A mobile device (smartphone with $3 \mathrm{G} / 4 \mathrm{G}$ connection, tablet with internet connection, and so on) with Android (version 4.3 or newer) or iOS (version 10.0 or newer) allows the electronic module to be configured and uses identifying information to associate the patient with the medical device. After this configuration process, all data will be recorded and sent to the mobile device via Bluetooth Low Energy (BLE) V4.0 or higher.

The data acquired by the electronic module are stored in the VS medical device and transmitted to the mobile device, which will encrypt the data and transfer them in real time to a dedicated certified health server. The information stored in the medical device itself is also encrypted and is recorded in a local memory operating system in a first-in, first-out manner. When there is no BLE link between the VS device and the mobile device (due to battery depletion or disruption of device pairing), the VS stores the data in its internal memory and automatically repeats the BLE pairing process (via a thread) with the mobile device until it succeeds. The memory of the VS can store data for up to 4 days. When pairing is operational again, the data acquired in real time are transmitted again and become visible on the mobile device (priority data); the data stored in the memory of the VS (resulting from the link break) are parallelised (via a thread) and sent directly to the buffer zone of the mobile device before being transferred to the certified health server.

Raw data and clinical parameters calculated via the VS will be concealed from caregivers in order not to influence care and will be analysed a posteriori from the cloud server. The ability to view the data in real time using the connection between the VS and the mobile device will not be used in this study.

\section{Intervention}

Patients meeting the study inclusion criteria a priori will be identified on the basis of the surgical programme and the elements collected during anaesthesia consultations. Two physicians (SM and SA), collaborators on the study, will meet these patients either the day before the operation or the same morning. They will present the study to the patients and answer any questions that may arise. The patients will decide whether to participate in this study after a period of reflection that they consider sufficient.

After written informed consent is obtained, the skin patch will be placed on the upper part of the sternum (figure 1).
All recorded data will include an absolute timestamp, where the mobile device is the reference.

Anaesthesia will be induced following a standard protocol with standard monitoring, including electrocardiography, non-invasive arterial blood pressure, pulse oximetry, capnography and inspiratory and expiratory sevoflurane concentration measurements, as well as train-of-four monitoring (Aisys anaesthesia machine, Carescape Monitor B850, General Electric Healthcare, Chicago, Illinois, USA). After surgery, all patients will be transferred to the PACU, where the usual automated monitoring (electrocardiography, non-invasive arterial blood pressure, pulse oximetry) will be performed and treatment will be administered.

The study will end when the patient leaves the PACU and returns to the surgical ward.

\section{Data collection}

Patient characteristics will be collected on inclusion in the study and will consist of age, sex, American Society of Anesthesiologists classification, body mass index, underlying diseases and classification of chest hair. Surgical indication, type of surgical procedure, procedural duration and eventual complications will be collected at the end of the study from the surgical and anaesthetic records.

All intraoperative monitoring variables (blood pressure, heart rate, pulse oximetry, ventilatory variables, including tidal volume, ventilatory frequency, peak and mean airway pressures and partial tension of end-tidal carbon dioxide pressure) will be collected using a Centricity Anaesthesia system at a rate of one value per minute. This system is an anaesthesia information management system that automatically collects and stores data in a repository, which can be subsequently exported as a spreadsheet file (GE Healthcare, Buc, France). All variables monitored in the PACU (blood pressure, heart rate derived from ECG, pulse oximetry and respiratory rate measured by thoracic impedance) will also be collected using a Centricity Anaesthesia system at one value per minute, except for arterial pressure, which will be measured at a lower frequency (from one measurement per minute to one every 15 min according to the clinical state of the patient). The data from the patch sensor will not be communicated to the anaesthesiologists, the nurses or other healthcare providers during the study period.

Because the main goal of this study is to investigate how postoperative physiological changes can be monitored with the patch, notes about any relevant findings will be made during the study. For example, if a complication occurs, it will be noted with the corresponding date and time and will be linked with the corresponding measurements.

Finally, when a nurse removes the patch, he/she will assess the status of the skin on the following scale: healthy skin (stage 0 ), redness limited to the contact area between the device and the skin (stage 1), redness extending beyond the contact surface of the device (stage 2) or the appearance of blisters (stage 3 ). The patient will be asked 
to evaluate his or her acceptance of the sensor using a 4 -point Likert scale $(0=$ intolerable, $1=$ very unpleasant, $2=$ slightly unpleasant, $3=$ no problem at all).

\section{Outcome measures}

Following the proposal of van Lier et $a l,{ }^{17}$ the validity of the wearable device will be assessed at three levels: (1) the raw signal level, based on the similarity of the two complete time series issued from the wearable device and from the reference device; (2) the clinical parameter level, comparing the values of blood pressure, heart rate, oxygen saturation and RR interval, averaged over a given time frame (5 min); and (3) the clinical event level, with the detection of relevant physiological changes, such as hypotension or hypopnoea, according to prespecified thresholds. The main objective is to determine the level of agreement between the parameters collected by the conventional monitor and the patch sensor (blood pressure, heart rate, respiratory rate and oxygen saturation) during patients' stay in the PACU.

The secondary objectives are (1) to determine the level of agreement of the measured parameters during anaesthesia; (2) to determine the frequency of artefacts and blank/null outputs from the wearable device and, more globally, the signal-level validity; (3) to estimate the diagnostic performance of the patch sensor at the event level, (4) to evaluate the influence of patient characteristics (gender, age, chest hair and body mass index) on the agreement between the two systems, (5) to identify any adverse events, and (6) to determine the acceptability of this patch to patients during their stay in the PACU.

\section{Statistical analysis}

Number of patients to be included

The aim of this study is to test the equivalence of two devices in recording the same data for the same patients. There is no standard method for the analysis of discrete time series (raw signal level). Therefore, an approach based on the quality of physiological data recorded (clinical parameter level) was used to calculate the required number of patients.

For heart rate equipment, the recommendations for the limits of acceptable error (boundaries of the BlandAltman plot) are a difference of \pm 5 beats per minute (bpm) or $\pm 10 \%$, whichever is greater, between the device of interest and a reference device, as proposed by the Association for Advancement of Medical Instrumentation in $2002 .{ }^{24}$ On that basis, we adopted these relative limits $( \pm 10 \%)$ for all parameters. We extracted possible values for the distribution of differences between a patch and a reference sensor from the papers of Smolle $e t a l,{ }^{25}$ Breteler $e t a l^{26}$ and van Lier $e t a l .{ }^{17}$ Two methods are considered to be in agreement when a predefined maximum allowed difference $(\Delta)$ is larger than the higher observed limit of agreement (LoA) and $-\Delta$ is smaller than the lower LoA. The $95 \%$ CI of the LoA must be taken into account for proper interpretation. Thus, in order to be $95 \%$ certain that the methods do not disagree, $\Delta$ must be larger than the upper $95 \%$ CI bound of the higher LoA and $-\Delta$ must be smaller than the lower $95 \%$ CI bound of the lower LoA. We then followed the new method proposed by Lu et al that takes power into account. ${ }^{27}$ Thus, assuming an SD of difference in heart rate of $4 \mathrm{bpm}$, a limit of acceptable error of $10 \mathrm{bpm}$ (ie, 2.5 times the SD), a two-sided alpha of $5 \%$ and a power level of $90 \%$, a sample size of 136 pairs of measures is required. If two measures $(m=2)$ of the same parameter are sampled in the same patient by two devices instead of one, the inclusion of $\mathrm{n}$ patients would yield $2 \mathrm{n}$ pairs of measures; however, taking into account the intrapatient correlation $r$, which is usually estimated to be 0.5 , the design effect is $1+(m-1) r=1.5$. Thus, the non-independence of observations within the same patient requires $1.5 * 2 *$ n paired measures to obtain the same amount of information as would be given by one pair of measures for each of $2 * n$ independent patients. Therefore, the need for 136 independent pairs indicates that $136 / 1.5=90$ patients need to be measured on two occasions by the two devices being assessed. This sample size is overestimated, since more than two measurement pairs could be obtained for each patient. However, it will also allow us (1) to analyse agreement at the clinical event level, those events being much less frequent than the sampling points, and (2) to perform an agreement analysis according to prespecified subgroups, defined by gender, age, body mass index and quantity of chest hair.

Taking into account that approximately $20 \%$ of the data may be unusable, it is anticipated that 115 patients need to be included in the study to ensure that the data of 90 patients (with two paired measurements, each in the postoperative period) can be analysed.

\section{Detection of artefacts}

A value will be automatically considered an artefact before data analysis if it is outside one of the 'normal' ranges defined in previous studies ${ }^{122-30}:$ (1) a value that is $>50 \%$ different from the previous value, unless it is followed by a value equal to $\pm 25 \%$; or (2) a value that is out of the physiologically plausible range (heart rate $<5$ or $>250 \mathrm{bpm}$, systolic artery pressure $<20$ or $>300 \mathrm{~mm}$ $\mathrm{Hg}$ or less than diastolic pressure plus $5 \mathrm{~mm} \mathrm{Hg}$, diastolic artery pressure $<5$ or $>225 \mathrm{~mm} \mathrm{Hg}, \mathrm{SpO}_{2}$ change of $\geq 8 \%$ between two consecutive measurements, respiratory rate $<3$ or $>60$ breaths per minute, skin temperature change of $\geq 1^{\circ}$ between two consecutive measurements). Furthermore, two clinicians will independently review all data in graphical form (one graph per variable per patient) before and after the artefacts are automatically identified. A third clinician will also review the data when there is discordance between the first two.

The selected rules to define artefacts may be updated according to experience. The adjusted rules will be recorded in a register, and all recordings will be reviewed in light of these new rules. 
Statistical analyses of reliability and agreement

Descriptive summaries will be provided for each parameter and for each device. For continuous variables, the mean, median and their $95 \%$ confidence limits, obtained using bootstrapping, will be provided. For discrete variables, counts, percentages and confidence limits, obtained using bootstrapping, will be provided. The relative frequency of data gaps and artefacts for each parameter will be given as a percentage of the total number of measurement points and of observations, respectively, with the corresponding 95\% CIs. The delay (hours) to the first occurrence of data loss or loss of device functionality will be described and analysed with Kaplan-Meier survival curves.

At the signal level (ie, for any physiological variable), cross-correlation will be used to compare the wearable device to a reference along the time series for each participant. If the cross-correlation coefficient is greater than 0.8 for all participants, the level of agreement will be deemed acceptable, and the assessment will be completed by calculating the differences. Complementarily, we will search for any systematic difference in mean or variance to correct the data from the wearable device.

At the parameter level, Bland-Altman analysis for repeated measurements, which accounts for multiple observations per individual, will be performed to create mean difference plots and compare the accuracy or bias (mean difference), precision (SD of difference) and the LoAs that are expected to contain $95 \%$ of paired differences between the measurements taken by the two methods (and their CIs), with those reported in the literature. A generalised linear mixed model will be used to calculate the components of variance, notably the withinsubject variation, to correct the variance of differences in this context of repeated measures. ${ }^{31} 32$ If the $95 \%$ CIs for the $95 \%$ LoAs are within the predefined agreement limits that are clinically acceptable, the two methods will be considered to have sufficient agreement to fulfil the agreement requirements.

In addition, a Clarke error grid analysis, with standard predetermined grids for heart rate, respiratory rate, artery pressure, $\mathrm{SpO}_{2}$ and temperature, will be conducted to identify the consequences of clinical decisions. $^{33}$

For adverse events, such as bradycardia, the sensitivity and specificity of the wearable device compared with the reference sensor will be calculated with $95 \%$ CIs.

Data from the postoperative period and the intraoperative period will be analysed separately.

A two-tailed $p$ value $<0.05$ will be considered statistically significant, without any adjustment for multiplicity. All statistical analyses will be performed using R software (V.3.2.4).

\section{Missing values}

Missing data will not be imputed.

\section{Data registration}

Data will be entered into the electronic case report form (eCRF) by trial or clinical personnel under the supervision of the trial site investigators at each participating centre. From the eCRF, the trial database will be established. The data collection process will be monitored by trained research coordinators.

\section{Patient withdrawal}

Any participant who wishes to terminate his/her participation in the study will be allowed to withdraw from the trial at any time without the need for further explanation. Participants who withdraw from the study will be followed up according to routine clinical practice.

\section{Safety}

Every serious adverse event (SAE) related to the studied procedure, regardless of whether it was expected, will be reported by the investigator to the sponsor within 24 hours on an SAE form that will list the date of occurrence, the criterion used to define severity, the intensity, the relationship with the study and the outcome. The period in which SAEs should be reported will last from the day written informed consent is obtained to the end of the follow-up period. Whenever an SAE persists at the end of the study, the investigator will follow the patient until the event is considered resolved. The management of SAEs will follow regulations and good clinical practices.

\section{Data handling and retention}

The data will be handled according to French laws under the responsibility of the Research Unit, Centre MédicoChirurgical Ambroise Paré (Neuilly-sur-Seine, France). All original records (including consent forms, reports of suspected unexpected serious adverse reactions and relevant correspondence) will be archived at the trial sites for 15 years. The cleaned and frozen trial database files will be anonymised and stored for 15 years.

\section{Patient and public involvement}

Patients and the public will not be involved in any phase of this study.

\section{Limitations}

A limitation of this study is that we will compare only minute-by-minute data. More sophisticated technologies that can be used to detect artefacts when monitoring trends in intensive care are as follows: (1) the Rosner statistic; (2) slope detection with rules; and (3) comparisons with a running median (median detection). ${ }^{34}$

Some categories of patients will not be included in the study: pregnant or breastfeeding women will be excluded because of French regulatory constraints, and patients older than 85 years will be excluded because they frequently present tremors, a well-known cause of artefacts.

Validation of the device in this study, where patients were monitored during their anaesthesia and PACU stays, does not allow us to generalise the possible favourable 
results to other situations. For example, such results could not be generalised to the postoperative period in the ward, where the risk of artefacts is elevated due to the patients' increased mobility. Similarly, it will be necessary to specifically study very elderly patients, given the frequency of tremors in that population, as tremors can be a source of artefacts.

\section{ETHICS AND DISSEMINATION \\ Ethics}

Ethics approval for this patch validation trial was obtained from the Ethical Committee (Toulouse, France) on 10 April 2020. Written informed consent will be required from patients prior to their participation in the study. The patch validation trial is registered at ClinicalTrials.gov.

We are not yet recruiting subjects for this study.

\section{Dissemination}

The Strengthening the Reporting of Observational Studies in Epidemiology statement (checklist of items that should be included in reports of cohort studies) will be followed.

\section{Publication plan}

Scientific presentations and reports derived from the study will be written under the responsibility of the coordinating investigator of the study with the approval of the principal investigators and the methodologist. The coauthors of the report and publications will be the investigators and clinicians involved, in proportion to their contributions to the study, as well as the biostatistician and associated researchers. The international recommendations for authorship will be followed.

\section{Author affiliations}

${ }^{1}$ Department of Anesthesiology, Hôpital Foch, Suresnes, France

${ }^{2} \mathrm{ICU}$, Clinique Ambroise Paré, Neuilly-sur-Seine, France

${ }^{3}$ Department of Information Systems, Hôpital Foch, Suresnes, France

${ }^{4}$ Research Unit, Clinique Ambroise Paré, Neuilly-sur-Seine, France

${ }^{5}$ Methodology Unit, GIRCI-IdF, Paris, France

${ }^{6}$ U1018 (Center for Epidemiology and Population Health), Paris-Saclay University, UVSQ, INSERM, Villejuif, France

Acknowledgements We would like to thank the person who made the figure 1 possible.

Contributors MLG, PS, PA, MM and MF contributed to the conception and design of the research protocol. SM, SA and BT provided critical input pertaining to the design of the trial interventions and procedures. MLG, PS, PA, MM and MF made substantial contributions to the interpretation of the data. PA designed the statistical analysis protocol. MF wrote the first draft of the protocol and this manuscript. All authors (MLG, PS, SM, SA, BT, MM, PA and MF) critically revised and modified the protocol and the article. All authors approved the final version to be published. All authors have agreed to be accountable for all aspects of the work and to ensure that questions related to the accuracy or integrity of any part of the work are appropriately investigated and resolved.

Funding This work is sponsored by the Research Unit, Centre Médico-Chirurgical Ambroise Paré, Neuilly-sur-Seine, France. All the study-related costs are borne by the promoter of the study (CMC Ambroise Paré, 27 Boulevard Victor Hugo, Neuilly-sur-Seine).

Competing interests None declared.
Patient and public involvement Patients and/or the public were not involved in the design, or conduct, or reporting, or dissemination plans of this research.

Patient consent for publication Obtained.

Provenance and peer review Not commissioned; externally peer reviewed.

Open access This is an open access article distributed in accordance with the Creative Commons Attribution Non Commercial (CC BY-NC 4.0) license, which permits others to distribute, remix, adapt, build upon this work non-commercially, and license their derivative works on different terms, provided the original work is properly cited, appropriate credit is given, any changes made indicated, and the use is non-commercial. See: http://creativecommons.org/licenses/by-nc/4.0/.

ORCID iDs

Pierre Squara http://orcid.org/0000-0003-1381-6773

Marc Fischler http://orcid.org/0000-0003-0729-5430

\section{REFERENCES}

1 Churpek MM, Edelson DP, Lee JY, et al. Association between survival and time of day for rapid response team calls in a national registry. Crit Care Med 2017;45:1677-82.

2 International Surgical Outcomes Study group. Global patient outcomes after elective surgery: prospective cohort study in 27 low-, middle- and high-income countries. Br J Anaesth 2016;117:601-9.

3 Michard F, Mountford WK, Krukas MR, et al. Potential return on investment for implementation of perioperative goal-directed fluid therapy in major surgery: a nationwide database study. Perioper Med 2015;4:11.

4 Ghaferi AA, Birkmeyer JD, Dimick JB. Variation in hospital mortality associated with inpatient surgery. N Engl J Med 2009;361:1368-75.

5 Morgan RJM, Williams F, Wright M. An early warning scoring system for detecting developing critical illness. Clin Intensive Care 1997;8:100.

6 Royal College of Physicians. Resources to support the adoption of the National early warning score. Available: https://improvement. nhs.uk/documents/3657/Resources_to_support_the_adoption_of_ NEWSFINAL.PDF

7 NHS. Available: https://www.england.nhs.uk/ourwork/clinicalpolicy/sepsis/nationalearlywarningscore/news-frequently-askedquestions/\#4-why-has-a-news2-been-launched

8 McGaughey J, Alderdice F, Fowler R, et al. Outreach and early warning systems (EWS) for the prevention of intensive care admission and death of critically ill adult patients on General Hospital wards. Cochrane Database Syst Rev 2007:CD005529..

9 Alam N, Hobbelink EL, van Tienhoven AJ, et al. The impact of the use of the early warning score (EWS) on patient outcomes: a systematic review. Resuscitation 2014;85:587-94.

10 Shamout F, Zhu T, Clifton L, et al. Early warning score adjusted for age to predict the composite outcome of mortality, cardiac arrest or unplanned intensive care unit admission using observational vital-sign data: a multicentre development and validation. BMJ Open 2019;9:e033301.

11 Mestrom E, De Bie A, Steeg Mvande, et al. Implementation of an automated early warning scoring system in a surgical ward: practical use and effects on patient outcomes. PLoS One 2019;14:e0213402.

12 Weenk M, Koeneman M, van de Belt TH, et al. Wireless and continuous monitoring of vital signs in patients at the general ward. Resuscitation 2019;136:47-53.

13 Michard F, Gan TJ, Kehlet H. Digital innovations and emerging technologies for enhanced recovery programmes. Br J Anaesth 2017;119:31-9

14 Beg MS, Gupta A, Stewart T, et al. Promise of wearable physical activity monitors in oncology practice. J Oncol Pract 2017;13:82-9.

15 Leth S, Hansen J, Nielsen OW, et al. Evaluation of commercial self-monitoring devices for clinical purposes: results from the future patient trial, phase I. Sensors 2017;17. doi:10.3390/s17010211. [Epub ahead of print: 22 Jan 2017].

16 Gillinov S, Etiwy M, Wang R, et al. Variable accuracy of wearable heart rate monitors during aerobic exercise. Med Sci Sports Exerc 2017;49:1697-703.

17 van Lier HG, Pieterse ME, Garde A, et al. A standardized validity assessment protocol for physiological signals from wearable technology: methodological underpinnings and an application to the E4 biosensor. Behav Res Methods 2020;52:607-29.

18 Breteler MJM, KleinJan EJ, Dohmen DAJ, et al. Vital signs monitoring with wearable sensors in high-risk surgical patients: a clinical validation study. Anesthesiology 2020;132:424-39. 
19 Leenen JPL, Leerentveld C, van Dijk JD, et al. Current evidence for continuous vital signs monitoring by wearable wireless devices in hospitalized adults: systematic review. J Med Internet Res 2020;22:e18636.

20 Wang R, Jia W, Mao Z. Cuff-free blood pressure estimation using pulse transit time and heart rate. 2014 12th International Conference on signal processing (ICSP), 2014:115-8.

21 Gholamhosseini H, Meintjes A, Baig M, et al. Smartphone-Based continuous blood pressure measurement using pulse transit time. Stud Health Technol Inform 2016;224:84-9.

22 Zhang Q, Zhou D, Zeng X. Highly wearable cuff-less blood pressure and heart rate monitoring with single-arm electrocardiogram and photoplethysmogram signals. Biomed Eng Online 2017;16:23.

$23 \mathrm{Li} \mathrm{G}, \mathrm{Yu}$ Y, Zhang C, et al. An efficient optimization method to improve the measuring accuracy of oxygen saturation by using triangular wave optical signal. Rev Sci Instrum 2017;88:093103.

24 Association for the Advancement of Medical Instrumentation. Cardiac monitors hrm, and alarms. Arlington, VA: American National Standard (ANSI/AAMI EC13: 2002), 2002: 1-87.

25 Smolle K-H, Schmid M, Prettenthaler H, et al. The accuracy of the

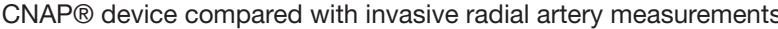
for providing continuous noninvasive arterial blood pressure readings at a medical intensive care unit: a Method-Comparison study. Anesth Analg 2015;121:1508-16.

26 Breteler MJM, Huizinga E, van Loon K, et al. Reliability of wireless monitoring using a wearable patch sensor in high-risk surgical patients at a step-down unit in the Netherlands: a clinical validation study. BMJ Open 2018;8:e020162.

27 Lu M-J, Zhong W-H, Liu Y-X, et al. Sample size for assessing agreement between two methods of measurement by Bland-Altman method. Int J Biostat 2016;12. doi:10.1515/ijb-2015-0039. [Epub ahead of print: 01 Nov 2016].

28 Morillo DS, Gross N. Probabilistic neural network approach for the detection of SAHS from overnight pulse oximetry. Med Biol Eng Comput 2013;51:305-15.

29 Cunningham S, Symon AG, Mclntosh N. The practical management of artifact in computerised physiological data. Int $J$ Clin Monit Comput 1994;11:211-6.

$30 \mathrm{Kool} \mathrm{NP,} \mathrm{van} \mathrm{Waes} \mathrm{JAR,} \mathrm{Bijker} \mathrm{JB,} \mathrm{et} \mathrm{al.} \mathrm{Artifacts} \mathrm{in} \mathrm{research} \mathrm{data}$ obtained from an anesthesia information and management system. Can J Anaesth 2012:59:833-41.

31 Bland JM, Altman DG. Agreement between methods of measurement with multiple observations per individual. J Biopharm Stat 2007;17:571-82.

32 Myles PS, Cui J. Using the Bland-Altman method to measure agreement with repeated measures. Br J Anaesth 2007;99:309-11.

33 Clarke WL, Cox D, Gonder-Frederick LA, et al. Evaluating clinical accuracy of systems for self-monitoring of blood glucose. Diabetes Care 1987;10:622-8.

34 Jakob S, Korhonen I, Ruokonen E, et al. Detection of artifacts in monitored trends in intensive care. Comput Methods Programs Biomed 2000;63:203-9. 